\title{
Collisional and resonance absorption of electromagnetic waves in a weakly collisional, inhomogeneous magnetoplasma slab
}

\author{
F. Rawwagah ${ }^{1}$, Manal M. Al-Ali ${ }^{1}$, A. M. Al-Khateeb ${ }^{1}$ and M. S. Bawa' aneh $^{1,2}$ \\ ${ }^{1}$ Department of Physics, Yarmouk University, Irbid, 211-63, Jordan. \\ ${ }^{2}$ Curriculum and Assessment Sector, Ministry of Education, United Arab Emirates. \\ Corresponding author: F. Rawwagah (e-mail: frawwag@yu.edu.jo)
}

\begin{abstract}
Absorbance of normally incident electromagnetic wave on a cold, weakly collisional, and inhomogeneous magnetoplasma slab is investigated. The plasma density is Budden-like sinusoidal profile, where the inhomogeniety is treated as a multilayered system of homogeneous sub-cells within the transfer matrix technique. For incident wave frequencies much above the ion cyclotron frequency, only right hand circularly polarized waves are relevant for wave propagation parallel to a static magnetic field. Calculations are performed in normalized parameters, that make the results suitable for many applications including atmospheric and laboratory plasmas. The presence of the dc-magnetic field leads to the formation of two absorption bands explained by plasma collisional dissipation and electron cyclotron resonance in the low frequency branch of the $R$-wave below the electron cyclotron frequency. The transmittance shows the emergence of the low frequency electron cyclotron wave, which becomes a Whistler mode at very low frequency. More detailed discussion on the effect of plasma collisionality, inhomogeneity, and dc-magnetic field on the propagation characteristics is given at the relevant place within the body of the manuscript.
\end{abstract}

INDEX TERMS Magnetoplasma slab, Inhomogeneous plasma, Reflectance, Transmittance, Absorbance

\section{INTRODUCTION}

T HE interaction of electromagnetic waves (EM-Waves) with a plasma slab has drawn the attention of researchers for decades for their wide applications in several fields. Recently, the propagation of EM-waves in cold plasma has gained a major interest due to the vast applications of atmospheric pressure plasma as reflectors or absorbers of EMwaves, which plays a dominant role in many applications that include stealth plasma technology [1]-[4], reentry blackout [5]-[10], communication and space plasma [11]-[15] as well as industrial material switches, absorbers and filters [16][20].

The behavior of an EM-wave in plasma is controlled by the wave parameters, such as the incident wave frequency, angle of incidence and wave polarization, and by the plasma parameters such as the electron density, electron temperature, thickness of the slab, frequency of collisions, and the magnetization state. Power transmittance (T), reflectance (R), and absorbance (A) of EM-waves incident on a plasma slab were numerically calculated for different conditions of incident waves and the characteristics of plasma slab.

Seikai et.al. studied the reflection and transmission of
EM-waves obliquely incident on an inhomogeneous moving magnetoplasma slab parallel to a magnetic field and found an evident correlation between the coefficient of reflection and the thickness and the velocity of the moving slab [21]. Lacina et.al. studied numerically the resonant interaction of obliquely incident EM-waves on an inhomogeneous plasma slab and found that a part of the incident electromagnetic energy is transformed into heat and the remaining part into energy of oscillation in the direction of the density gradient [22]. Lin Lie et.al. discussed the dependence of R, T, and $\mathrm{A}$ of a plane EM-wave that is normally incident on a homogeneous plasma slab and found that only waves with certain wavelengths can be absorbed, reflected or transmitted. The increase of collision frequency of the plasma increases the absorbance and decreases the transmittance and the reflectance [23].

Highly collisional and high density plasma with partially linear and sinusoidal electron density profile are found to significantly absorb the EM-wave energy over a wide frequency band with greatly improved absorption characteristics for slabs with higher thickness [24]. Matrix formalism of the problem of EM-wave propagation in dielectric media [25] 
can be utilized to overcome the inhomogeneity in the plasma slab medium. Properties of great influence on the propagation characteristics, such as the medium inhomogeneity, can be modeled by replacing an inhomogeneous layer by a succession of thin discrete homogenous sub-layers [1], [3], [26]. In this paper we investigate the propagation characteristics of a normally incident electromagnetic wave on a cold, weakly collisional, and inhomogeneous magnetoplasma slab. A Budden-like sinusoidal density profile is considered and the plasma slab is treated as a multilayered system of homogeneous sub-slabs within the Max-Born transfer matrix technique [11], [27]. Frequencies of interest are those frequencies much above the ion cyclotron frequency so that only the motion of electrons is considered. Consequently, only the right-hand, circularly polarized wave is relevant for wave propagation parallel to a static magnetic field. Calculations are performed in normalized parameters, that make the results suitable for many applications including atmospheric as well as laboratory plasma. The paper is organized as follows: In section II we present the model equations used in our numerical calculations. In section III we present numerical results, and finally, in section IV we discuss the main results and conclusions.

\section{MODEL EQUATIONS}

The geometry of the plasma slab is shown in Fig. 1. We assume that waves are normally incident from the left semiinfinite medium (vacuum) into the bound plasma slab of width $a$. The dielectric permittivity of input (left) and output (right) semi.infinite regions are $\epsilon_{\ell}$ and $\epsilon_{r}$, respectively.

Assume a linearly polarized plane wave such that $\vec{E}(x, t)=\hat{z} E_{z}(x, t)$ is incident on an inhomogeneous magnetoplasma slab extending from $x=0$ to $x=a$. The incident, reflected and transmitted waves are propagating along the $x$-axis parallel to a background uniform magnetostatic field $\vec{B}=\hat{x} B_{0}$, as shown in Figure 1 .

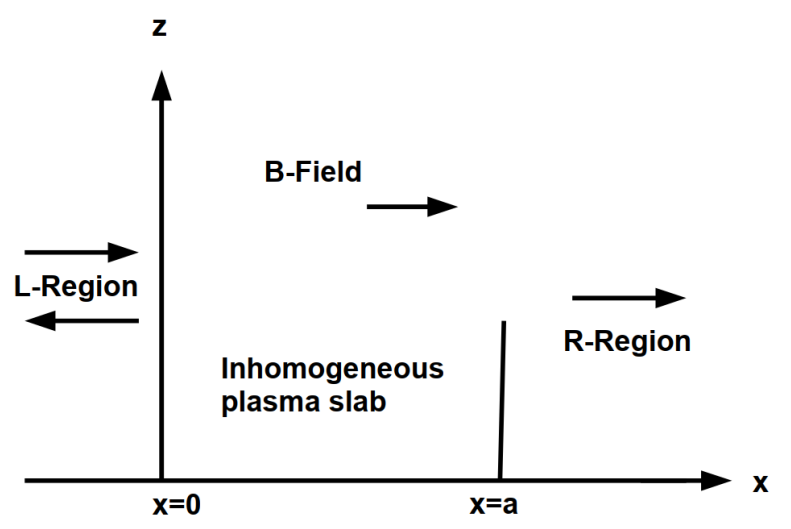

FIGURE 1: Bound inhomogeneous plasma slab in a dc-magnetic field.

We divide the inhomogeneous plasma slab into $N$ sufficiently thin homogeneous sub-slabs. The $m^{\text {th }}$ layer width is $d_{m+1}-d_{m}$, where $m=1,2,3, \ldots, N$. The electromagnetic fields at the inner face of the exit layer $m=N$ are related to the fields at the input interface of incidence by the global (overall) transfer matrix $M$ of the whole multilayered structure [25], [27], namely,

$$
\begin{aligned}
& \left(\begin{array}{l}
E \\
B
\end{array}\right)_{x=0}=M\left(\begin{array}{l}
E \\
B
\end{array}\right)_{x=N d}, \\
& M=\prod_{m=1}^{N} M_{m}=\left(\begin{array}{ll}
M_{11} & M_{12} \\
M_{21} & M_{22}
\end{array}\right) \\
& M_{m}=\left(\begin{array}{cc}
\cos \delta_{m} & \frac{i}{\gamma_{m}} \sin \delta_{m} \\
i \gamma_{m} \sin \delta_{m} & \cos \delta_{m}
\end{array}\right),
\end{aligned}
$$

where $M_{m}$ stands for the $m^{\text {th }}$ sub-slab transfer matrix which connects the electromagnetic fields at one interface with those at the other, and $\delta_{m}$ is the phase difference corresponding to one traversal of the $m^{\text {th }}$-layer. For normal incidence, the difference between TM and TE polarized waves disappears, and therefore, the parameters $\gamma_{m}$ and $\delta_{m}$ are given by

$$
\begin{gathered}
\gamma_{m}=\sqrt{\mu_{m} \epsilon_{m}}=\sqrt{\mu_{0} \epsilon_{0}} \sqrt{\varepsilon_{m}}, \\
\delta_{m}=\omega d_{m} \sqrt{\mu_{m} \epsilon_{m}}=\omega d_{m} \sqrt{\mu_{0} \epsilon_{0}} \sqrt{\varepsilon_{m}},
\end{gathered}
$$

where $\omega$ is the angular frequency of the incident wave, $\mu_{m}$, $\epsilon_{m}, d_{m}, \varepsilon_{m}$ are, respectively, the $m^{t h}$ sub-slab permeability, permittivity, width, and dielectric function. All necessary information to characterize the propagation of electromagnetic waves across the inhomogeneous plasma slab is contained in equations (1-5).

The matrix $M$ is the transfer matrix of the whole multilayered system, and its matrix elements determine the transmission and the reflection amplitudes for the entire system. The complex transmission $\tilde{\tau}$ and reflection $\tilde{\rho}$ coefficients in terms of global transfer matrix elements are [27],

$$
\begin{aligned}
\tilde{\tau} & =\frac{2 \gamma_{\ell}}{\gamma_{\ell} M_{11}+\gamma_{\ell} \gamma_{r} M_{12}+M_{21}+\gamma_{r} M_{22}}, \\
\tilde{\rho} & =\frac{\gamma_{\ell} M_{11}+\gamma_{\ell} \gamma_{r} M_{12}-M_{21}-\gamma_{r} M_{22}}{\gamma_{\ell} M_{11}+\gamma_{\ell} \gamma_{r} M_{12}+M_{21}+\gamma_{r} M_{22}}, \\
T & =\frac{\sqrt{\varepsilon_{r}}}{\sqrt{\varepsilon_{\ell}}} \tilde{\tau} \tilde{\tau}^{*}, \quad R=\tilde{\rho} \tilde{\rho}^{*},
\end{aligned}
$$

where $R$ and $T$ are the corresponding reflectance and transmittance. The absorbance (A) is defined as $A=1-$ $R-T$.

\section{NUMERICAL RESULTS}

In the following numerical investigation, we assume an inhomogeneous plasma slab density that may be suitable for ionospheric applications [11], [24].

$$
n_{e}(x)= \begin{cases}n_{0 e} \sin \frac{\pi x}{a} & 0 \leq x \leq \frac{a}{2} \\ n_{0 e}\left[1-\sin \frac{\pi}{a}\left(x-\frac{a}{2}\right)\right] & \frac{a}{2} \leq x \leq a\end{cases}
$$


Here $n_{0 e}$ is the peak density of plasma electrons. According to equation (9) the plasma density increases gradually for zero level (no plasma) at $x=0$ and increases until it reaches its peak value at the center of the slab $a / 2$, then it decreases gradually until it disappears completely at $x=a$. The $5 \lambda$ wide slab was divided into 50 sub-slabs, where the plasma density function of equation (9) is a smooth function.

For high frequency waves, the plasma ions are assumed to be immobile neutralizing background of positive charge immersed in the uniform magnetostatic field $\vec{B}_{0}=B_{0} \hat{x}$. For wave propagation along the applied magnetic field, a magnetoplasma of cold electrons and cold ions supports two transverse right and left circularly polarized electromagnetic waves. Since left circularly polarized waves are off-resonant with the electron motion, only right polarized waves rotating with the same sense as the electrons will be considered. The $R$-wave has two pass bands separated by a stop band, namely, $0<\omega<\omega_{c e}$ and $\omega>\omega_{R}$. Here $\omega_{R}$ is the cutoff frequency of the $R$-wave given by

$$
\omega_{R}=\frac{\omega_{c e}}{2}+\frac{1}{2} \sqrt{\omega_{c e}^{2}+4 \omega_{p e}^{2}}
$$

The $m^{t h}$ sub-slab dielectric function for the $R$-wave is

$$
\varepsilon_{ \pm}^{(m)}=1-\frac{\omega_{p e, m}^{2}}{\omega\left(\omega-\omega_{c e, m}-j \nu_{e}\right)}, \quad \omega_{p e, m}^{2}=\frac{e^{2} n_{e, m}}{\epsilon_{0} m_{e}}
$$

where $\nu_{e}$ is the electron collision frequency with neutral particles. The electrical properties of the plasma regime are embedded within the dielectric function [28]-[31].

In all numerical examples presented below, we use normalized quantities and therefore, we initiate the calculations by an arbitrary incident wave frequency $\omega=\omega_{0}$. In all calculations we adopt the normalized width of the plasma slab $a / \lambda_{0}=5$. The normalized electron cyclotron frequency $\omega_{c e}$ in each plasma slab can take one of the values $\omega_{c e} / \omega_{p e}=$ $0,0.2,0.6,1,1.4$, and the normalized collision frequency of electrons with neutrals $\nu_{e} / \omega_{p e}=0.01$ for weak but relevant collisional damping. High collision frequencies add nothing to the understanding of the general problem.

For obvious characterization of wave propagation in an inhomogeneous magnetoplasma slab, numerical results can be easily understood by selecting the relative electron density to be the independent variable, namely, $\sqrt{n_{0 e} / n_{c}}=\frac{\omega_{p e}}{\omega_{0}}=$ $\frac{\omega_{p e} \lambda_{0}}{2 \pi c}$, where $\lambda_{0}$ is the free space wavelength and $n_{c}$ is the plasma critical density for a plane wave incident on an unmagnetized cold homogenous plasma. Accordingly, the plasma density increases by moving to the right along the $\omega_{p e}$ coordinate at fixed wave frequency $\omega_{0}$. Similarly, the wave frequency increases as we move to the left at a fixed plasma density.

In Figs. 2 and 3 we present $R, T$ and $A$ for weakly collisional, unmagnetized plasma slabs for the homogeneous and inhomogeneous cases, respectively. For a homogeneous plasma slab (Fig.2) the incident wave is mainly reflected at the critical plasma density $\omega_{0}=\omega_{p e}$, while the main reflection point diffuses to the right for the inhomogeneous slab occuring at frequencies slightly above $\omega_{p e}$. The oscillations in $R, T$ and $A$ curves of Fig.2 result from the in-plasma interferences between waves reflected from the inner sides of the plasma interfaces located at $x=0$ and $x=a$. For the homogeneous slab (Fig.3) we observe that the oscillations of the in-plasma interferences are smeared out by the slab inhomogeneity with a slight diffusion of the reflection point to the right of $\omega_{p e}$ as mentioned above. In addition, the curves of Fig. 3 show that the absorbance from collisional dissipation becomes broader due to the slab inhomogeneity.

In Figs. 4 and 5 we plot the reflectance $R$ for weakly collisional magnetoplasma slabs for different normalized cyclotron frequencies for the homogeneous and inhomogeneous cases, respectively. For non-vanishing cyclotron frequency, we observe a development of the reflection bands, which become narrower with increasing cyclotron frequency. For $\omega_{c e}=1.4 \omega_{p e}$, the reflection band is in the frequency range $\omega_{p e}<\omega_{c e}<\omega_{0}$. This condition implies that waves are reflected either at low plasma frequencies for fixed $\omega_{0}$, or equivalently at higher frequencies for fixed $\omega_{p e}$.

By increasing the normalized plasma collision frequency, the oscillations of in-plasma interferences in Fig.4 will be washed out. Accordingly, extending the range of wave propagation (right shift of reflection point) and the removal of the oscillations of in-plasma interferences is either due to collisionality or inhomogeneity of the plasma slab.

In Fig.6 we plot the transmittance $T$ for weakly collisional magnetoplasma slabs for different normalized cyclotron frequencies. For either a low density plasma or a high incident wave frequency the plasma is initially transparent, and the wave transmission decreases until it vanishes at cutoff. Within the stop band the transmittance is zero, where the incident wave becomes an evanescent wave, and there will be no wave propagation for a complex plasma index of refraction and complex wave number.

At the right boundary of the stop band we observe the emergence of a propagating mode from the stop band which becomes obvious for thinner stop bands when the cyclotron frequency is increased relative to the plasma frequency $\omega_{c} \geq$ $\omega_{p e}$. This mode is the well known low-frequency electron cyclotron wave, which is an important characteristic of the dispersion relation of right hand circular electromagnetic waves (R-wave) in magnetoplasma for parallel propagation.

In Fig. 7 we plot the Absorbance $A$ for weakly collisional magnetoplasma slabs for different normalized cyclotron frequencies. The curves for $\omega_{c e} \neq 0$ show two absorption bands; the left absorption band peaks around the corresponding cutoff frequency for each $\omega_{c e}$ value. These absorption bands are of collisional nature due to dissipation in the vicinity of the high frequency cutoff point $\omega_{R}$. The presence of the magnetic field enhances the collisional absorption peak making it narrower, and shifts it down to the high frequency (or to the low plasma density regions) $\frac{\omega_{p e}}{\omega_{0}}<1$. For unmagnetized plasma $\omega_{c e}=0$, the collisional absorption bands are centered 
around the left boundary of the stop band at $\omega_{0}=\omega_{p e}$.

The right absorption band of each curve appears for $\omega_{c e} \neq$ 0 and is related to the emergence of the propagating wave at the right boundaries of the stop band. For the cyclotron frequency $\omega_{c e}=1.4 \omega_{p e}$, the right absorption band is in the frequency region where the plasma frequency is below both the incident wave and electron cyclotron frequencies. For the cyclotron frequencies $\omega_{c e} \leq \omega_{p e}$, the right bands move into the low wave frequency or high plasma density regions.

The emerging propagating mode in the low branch of the $R$-wave is the electron-cyclotron wave which moves down from high frequency pass band through the stop band into the low frequency pass band. Accordingly, the wave approaches the resonance point from the incident direction at the end of the stop band (here from left), and obviously, the second absorption right bands are due to the electroncyclotron resonance at the elctron cyclotron frequency. The results agree with Budden analytical calculations, in which he showed that the wave energy is steadily build up and some is absorbed at the resonance, while some tunnel through to the other side with no reflection [11]. The wave energy tunneled to the other side, appear in the low frequency region which is finally attenuated by the plasma.

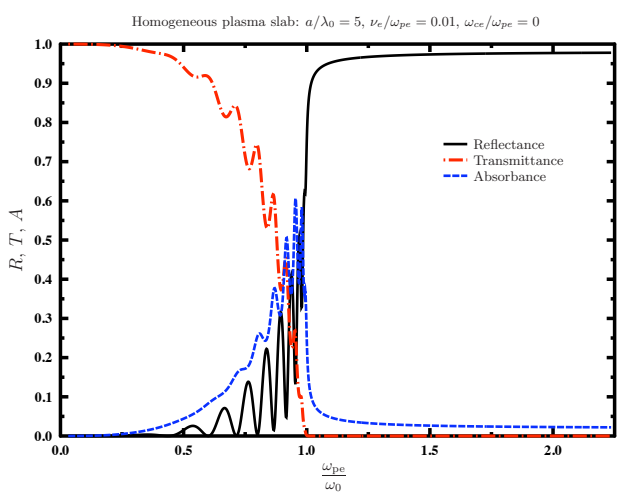

FIGURE 2: Reflectance, Transmittance, and Absorbance for a homogeneous unmagnetized plasma slab

\section{CONCLUSION}

In this paper we present a numerical investigation of the reflection, absorption and transmission of a transverse electromagnetic wave normally incident on an inhomogeneous, magnetized, and bound cold plasma slab. The effect of inhomogeneity of an unmagnetized plasma slab is to smooth out the R, T, and A curves eliminating the in-slab interference effects observed in the curves of the homogeneous slab. A similar effect is observed when collisions are introduced in the slab indicating that interference effects can be washed out either by inhomogeneity of the slab or by the plasma collisionality. Another effect of inhomogeneity is to broaden the absorbance peak of the unmagnetized slab. For wave propagation along a constant magnetic field, at high fre-

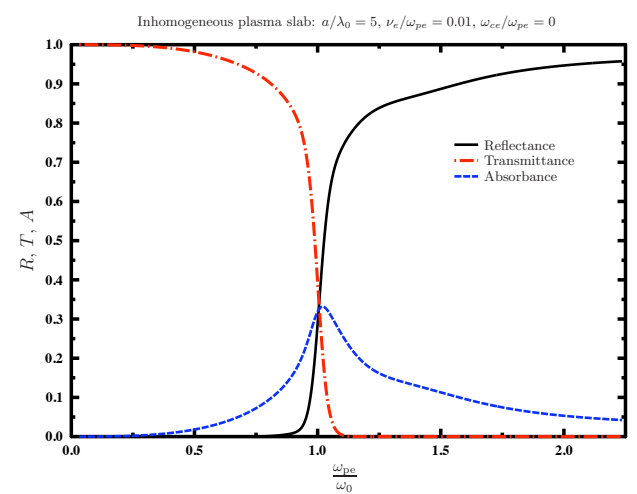

FIGURE 3: Reflectance, Transmittance, and Absorbance for an inhomogeneous unmagnetized plasma slab

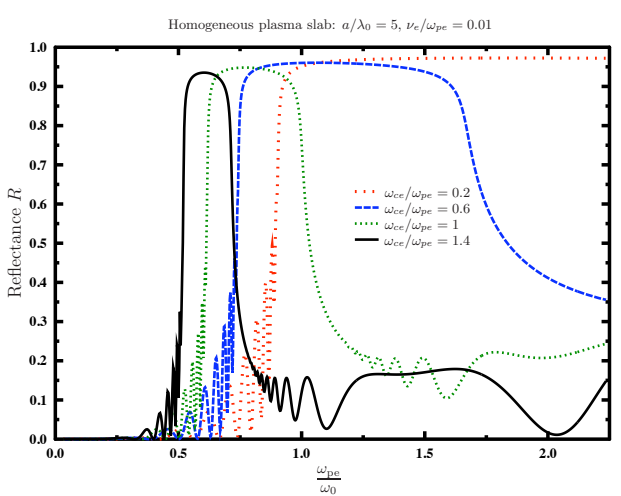

FIGURE 4: Reflectance for an homogeneous magnetoplasma slab

quencies, only transverse right circular mode is resonant with the electrons. Reflection bands develop and become narrower with the increase of the magnetic field. Waves are reflected either at low plasma frequencies for fixed frequency of incident waves, or equivalently at higher frequencies for fixed plasma frequency.

The presence of the dc-magnetic field leads to the formation of two absorbance (or reflection) bands, which are explained by plasma collisional dissipation and electron cyclotron resonance in the low frequency branch of the rightwave below the electron cyclotron frequency. The transmittance shows a low frequency electron cyclotron wave, that is transformed into a Whistler mode at very low frequencies.

The emerging propagating mode which moves down from high frequency pass band to the low frequency pass band passing through a stop band with the wave approaching the resonance point at the end of the stop band is consistent with Budden analytical calculations. 


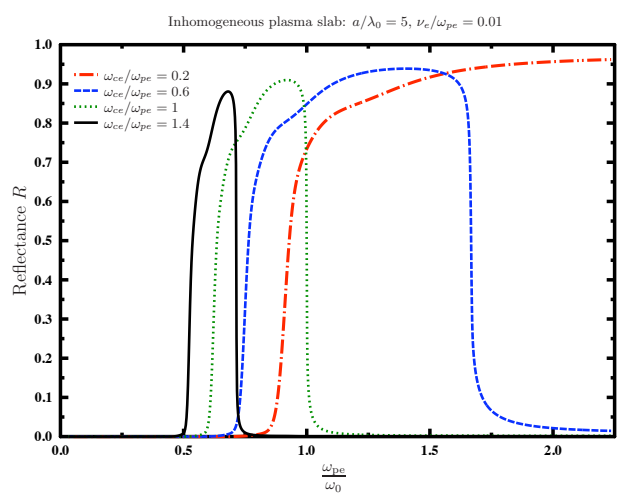

FIGURE 5: Reflectance for inhomogeneous magnetoplasma slab

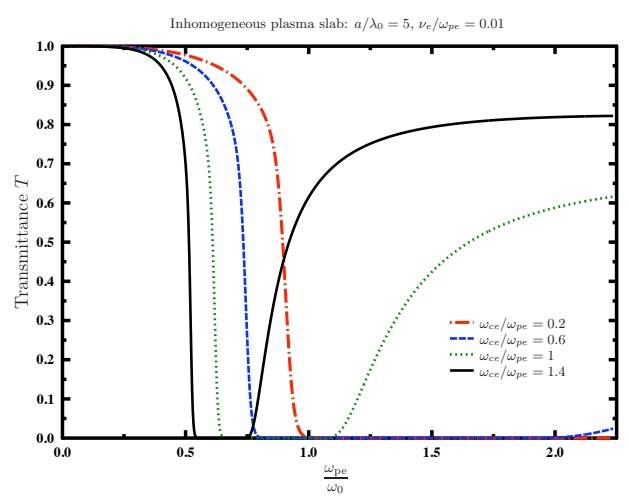

FIGURE 6: Transmittance for inhomogeneous magnetoplasma slab

\section{REFERENCES}

[1] Mounir Laroussi and J. Reece Roth, "Numerical calculation of the reflection, absorption and transmission of microwaves by a nonuniform plasma slab," IEEE Transactions on Plasma Science, vol. 21, pp.366-372 (1993).

[2] Mahdi Rahmanzadeh et. al, "Analytical Investigation of Ultrabroadband Plasma Graphene Radar Absorbing Structure", IEEE Transactions on Plasma Science," vol. 45, pp. 945-954, (2017).

[3] M. S. Bawa' aneh, A. M. Al-Khateeb and A. S. Sawalha, "Microwave propagation in a magnetized inhomogeneous plasma slab using the AppletonHartree magneto-ionic theory," Canadian Joural of Physics, vol. 90, pp.241-247 (2012).

[4] A. M. Al-Khateeb, M. A. Khasawneh, and M. S. Bawa'aneh, "Forward recursion approach of electromagnetic wave propagation characteristics in a slab of inhomogeneous magnetoplasma", Advanced Electromagnetics, vol. 8, pp. 44-50 (2019).

[5] J. P. Rybak, J. P. and R. I. Churchill, "Progress in reentry communications," IEEE Transactions on Aerospace \& Electronic Systems, vol. 7, pp. 879894, (1970)

[6] M. S. Bawa' aneh, A. M. Al-Khateeb and A. S. Sawalha, "Microwave Propagation in Warm, Collisional Magnetoionic Media," IEEE Transactions on Plasma Science, vol. 41, pp.2496-2500 (2013).

[7] Mohammad A Matin, "Review on Millimeter Wave Antennas- Potential Candidate for 5G", Advanced Electromagnetics, vol. 5, pp. 98-105 (2016).

[8] Xin Yang, Bing Wei and Weike Yin, "A new method to analyze the EM wave propagation characteristics in the hypersonic sheath", Optik, vol. 148, pp. 187-195 (2017).

[9] M. S. Bawa' aneh, A. M. Al-Khateeb, and Y.-C. Ghim, "Thermal Enhancement of Absorption of EM Radiation in a Hot Magnetoplasma Slab," IEEE Transactions on Antennas and Propagation, vol. 66, No. 12, pp. 6525-6530 (2018).

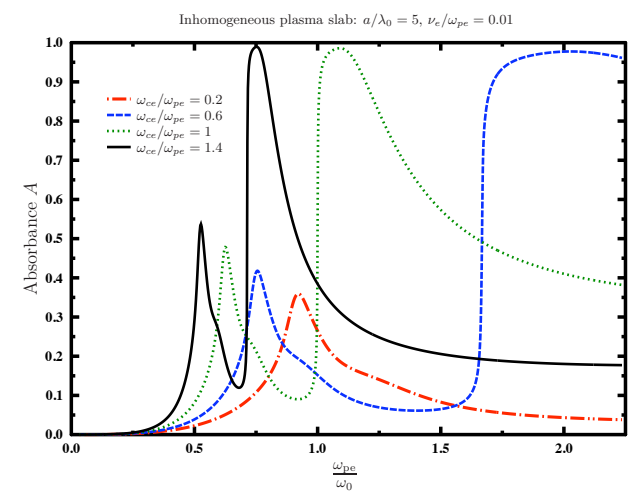

FIGURE 7: Absorbance for inhomogeneous magnetoplasma slab

[10] Kai Yuan, Jiamin Chen, Linfang Shen, Xiaohua Deng, Ming Yao, and Lujun Hong, "Impact of Reentry Speed on the Transmission of Obliquely Incident THz Waves in Realistic Plasma Sheaths", IEEE Transactions on Plasma Science, vol. 46, pp. 373-379 (2018).

[11] K. G. Budden, "Radio waves in the ionosphere", Cambridge university press (1961)

[12] M. Laroussi, "Interaction of microwaves with atmospheric pressure plasmas," Int. J. Infrared Millim. Waves, vol. 16, pp. 2069-2083 (1995).

[13] Yunxian Tian, Weizhong Yan, Xiaoliang Gu, Xiaolin Jin, Jianqing Li and Bin $\mathrm{Li}$, "Effect of magnetized plasma on the propagation properties of obliquely incident THz waves," AIP Advances, vol. 7, pp. 125325-11 (2017).

[14] Jiyun Lee, Y. T. Jade Morton, Jinsil Lee, Hee-Seung Moon and Jiwon Seo, "Monitoring and Mitigation of Ionospheric Anomalies for GNSS-Based Safety Critical Systems: A review of up-to-date signal processing techniques," IEEE Signal Processing Magazine, vol. 34, pp. 96-110 (2017).

[15] Cagatay Tanil; Samer Khanafseh; Mathieu Joerger; Boris Pervan, "An INS Monitor to Detect GNSS Spoofers Capable of Tracking Vehicle Position," IEEE Transactions on Aerospace and Electronic Systems, vol. 54, pp. 131143 (2018).

[16] M. K. Hedayati, et. al, "Photodriven Super Absorber as an Active Metamaterial with a Tunable Molecular Plasmonic Coupling," Advanced Optical Materials," vol. 2, pp. 705-710 (2014).

[17] M.A. Abdalla and Z. Hu, "On the study of development of $x$ band meta material radar absorber," Advanced Electromagnetics, vol. 1, no.3, pp. 94 98 (2012).

[18] F. Costa and A. Monorchio, "Electromagnetic absorbers based on high impedance surfaces: From ultra-narrow band to ultra-wide band absorption," Advanced Electromagnetics, vol. 1, no.3, pp. 7-12 (2012).

[19] Chen Zhou, Mehdi Keshavarz Hedayati and Anders Kristensen, "Multifunctional waveguide interferometer sensor: simultaneous detection of refraction and absorption with size-exclusion function", Optics Express, vol. 26, pp. 24372-24383 (2019).

[20] Aqeel H. Naqvi and Jeong-Heum Park, "Via-monopole based quasi YagiUda antenna for W-band applications using thorough glass silicon via (TGSV) technology", IEEE Access, vol. 8, pp. 9513-9519 (2020).

[21] S. Seikai, K. Tanaka, and T. Shiozawa, "Reflection and transmission of obliquely incident electromagneticwaves by an inhomogeneousplasma slab moving parallel to the magnetostatic field," Radio Science, vol. 9, pp. 403-408 (1974).

[22] J. Lacina and J. Preinhaelter, "The interaction of an electromagnetic wave with an inhomogenbous plasma slab," Czechoslovak Journal of Physics B, vol. 33, pp. 160-174 (1983).

[23] Lin Lie, Wu Bin, Wu Chengkang, "Propagation Properties of Electromagnetic Wave in a Plasma Slab," Plasma Science and Technology, vol. 5, pp. 1905-1908 (2003).

[24] C.S. Gurel and E. Oncu, "Interaction of electromagnetic wave and plasma slab with partially linear and sinusoidal electron density profile", Progress In Electromagnetics Research Letters, vol. 12, pp. 171-181 (2009).

[25] Frank L. Pedrotti, Leno M. Pedrotti, Leno S. Pedrotti, "Introduction to Optics”, 3rd Edition, Cambridge University Press (2017). 
[26] Bin Jie Hu, Gang Wei and Sheng Li Lai, "SMM Analysis of Reflection, Absorption and Transmission from Nonuniform, Magnetized Plasma Slab," IEEE Transactions on Plasma Science, vol. 27, pp.1131-1136 (1999).

[27] Max Born and Emil Wolf, "Principles of Optics: Electromagnetic Theory of Propagation, Interference and Diffraction of Light," seventh edition, Cambridge Press (2003).

[28] Thomas H. Stix, "Waves in Plasmas", AIP, New York (1992).

[29] D Gary Swanson, "Plasma Waves", 2nd Edition, IOP Publishing Ltd (2003).

[30] M. S. Bawa'aneh and T. J. M. Boyd, "Enhanced levels of stimulated Brillouin reflectivity from non-Maxwellian plasmas", Journal of Plasma Physics vol. 73, No. 2, pp. 159-166, 2007.

[31] Umran Inan and Marek Gokowski, "Principles of Plasma Physics for Engineers and Scientists", Cambridge University Press, New York (2011). 